\title{
Heterosis Analysis for Yield and its Components Traits in Pearl Millet [Pennisetum glaucum (L.) R. Br.]
}

\author{
S. H. Patil", P. B. Wadikar, A. R. Talape and S. L. Waghmode \\ Department of Agricultural Botany, College of Agriculture Latur, Vasantrao Naik \\ Marathwada Krishi Vidypeeth, Parbhani, (M.S), India \\ *Corresponding author
}

\section{A B S T R A C T}

Keywords

Heterosis,

Heterobeltosis,

Standard heterosis,

Pearl millet

Article Info

Accepted:

15 December 2020

Available Online:

10 January 2021
Pearl millet is the most important drought tolerant crop of tropical and subtropical region of the world. Pearl millet has balanced genetic load and show considerable inbreeding depression. Therefore, the varieties aimed to be developed in pearl millet should have heterozygous nature to be heterotic and at the same time homogenous constitution to be synchronous and uniformly productive. Therefore, breeding efforts are directed towards developing hybrids, synthetic and composite populations. The mid parent, better parent and standard heterosis were estimated for 10 yield and its contributing characters in pearl millet [Pennisetum glaucum (L.) R. Br.]. The analysis of variance disclose significant differences among treatments, parents and crosses for all characters studied, indicated the substantial amount of genetic variability among the treatments, parents and crosses in the experimental material used in present study. The crosses S-16/704 A x S-19/12, S-16/769 A x S-19/13, S-16/769 A x S-19/11, S-16/704 A x S-19/13 and DHLB-23 A x S-19/04 had significant average heterosis, heterobeltosis and standard heterosis over both standard checks viz., Aadishakti and AHB-1200 (Fe) for grain yield and most of the yield contributing traits. Hence, these crosses could be checked for their stable performance in multi location trials over environment.

\section{Introduction}

Pearl millet is the most important cereal crop of tropical and subtropical region of the world. It is predominantly consumed as a basic food grain and source of feed and fodder. It is staple food for billions of people living in harsh environments characterized by erratic rainfall and nutrient- poor soil and nutritionally superior. In fact, Pearl millet is the only suitable and efficient crop for arid and semi-arid conditions because of its efficient utilization of soil moisture and higher level of heat tolerance than sorghum and maize. Pearl millet grains contain 9-15\% protein, 5\% fat and 2-7 \% minerals and impart substantial energy to the body with easy digestibility. India is a major pearl millet producing country with 43.3 per cent of the world area and 42 per cent of world production. In the states of Rajasthan, Maharashtra, Gujarat, Madhya Pradesh, Karnataka, Andhra Pradesh, Uttar Pradesh and Tamil Nadu it is mainly cultivated. In 
terms of both area) 7.50 million ha) and production) 9.73 million ton), India is the largest producer of pearl millet with an average productivity is $850 \mathrm{~kg} / \mathrm{ha}$ ) Anonymous, 2018). Maharashtra is the fifth largest producer of pearl millet in India with $11.04 \%$ area and $8.21 \%$ production. Pearl millet shows considerable inbreeding depression therefore, the varieties aimed to be developed in pearl millet should have heterozygous nature to be heterotic and at the same time homogenous constitution to be synchronous and uniformly productive.

Therefore, breeding efforts are directed towards developing hybrids, synthetic and composite populations. For the development of effective heterosis breeding programme in pearl millet, having information about genetic architecture and estimated prepotency of parents in hybrid combinations is essential. This knowledge in fact helps in exploiting heterosis for commercial purpose.

\section{Materials and Methods}

The experimental material comprised of four male sterile lines S-16/760A, S-16/769A, S16/704A, DHLB-23A and ten restorers $S$ 19/03, S-19/04, S-19/05, S-19/08, S-19/09, S19/10, S-19/11, S-19/12, S-19/13, S-19/14 with two checks Aadishakti and AHB 1200) $\mathrm{Fe})$. Crosses were done in line $\mathrm{x}$ tester fashion to obtain 40 hybrids. All experimental material evaluated in randomized block design with two replication during Kharif 2019. Five competitive plants were selected randomly from each treatment in each replication for grain yield per plant and its component traits viz., Days to $50 \%$ flowering, Days to maturity, Number of effective tillers per plant, Plant height, Earhead length, Earhead girth, 1000 seed weight, Grain yield per plant, Fodder yield per plant, Harvest index and averages were worked out. The mean data was utilized for estimating the heterosis as per cent increase or decrease in mean value of $F_{1}$ hybrids over better parent and standard checks as per Fonseca and Patterson) 1968).

\section{Results and Discussion}

To test the significance of differences among the treatments analysis of variance of mean was carried out. The data pertaining to the analysis of variance) Table 1) revealed significant differences among treatment, parents and crosses for all the characters) except plant height for parent $v / s$ crosses) indicated the substantial amount of genetic variability among the treatments, parents and crosses in the experimental material used in present study.

Negative heterosis is desirable for days to $50 \%$ flowering. The hybrid S-16/760 A x S19/08) -10.53\%) exhibited highest significant and negative heterosis over mid parent and over better parent) 16.39\%). The crosses, DHLB-23 A x S-19/09 and DHLB-23 A x S$19 / 10$ recorded highest percentage of heterosis over standard checks; Aadishakti) 7.55\%) and AHB-1200) Fe) -9.26\%)) Table 2.). For the character days to maturity $S$ 16/760 A x S-19/09) -8.99\%) followed by S16/760 A x S-19/08) -8.79\%) and S-16/769 A x S-19/09) -7.43) exhibited highest negative heterosis in desirable direction over mid parent. The cross, S-16/760 A x S-19/08) 11.7) manifested highest percentage of negative heterosis over better parent. As regards to standard checks, the crosses S16/760 A x S-19/09, S-16/769 A x S-19/09 and DHLB-23 A x S-19/09 had exhibited highest negatively significant heterosis over Aadishakti) -8.99\%) and AHB-1200) Fe)) 7.95) respectively. These findings in which negative heterosis is desirable are in accordance with Pawar et al., (2015), Bhaskar et al., (2017). 
The heterosis over mid parent and better parent for number of effective tillers per plant ranged from -29.03) S-16/704 A x S-19/09) to 64.52 per cent) DHLB-23 A x S-19/13) and 38.89) S-16/704 A x S-19/09) to 59.37 per cent) DHLB-23 A x S-19/13), respectively. The cross DHLB-23 A x S-19/13 exhibited highest percentage of heterosis over mid parent) $64.52 \%$ ) and better parent) $59.37 \%$ ) followed by DHLB-23 A x S-19/05) 54.29\% and $42.11 \%$ ) respectively. The hybrids, DHLB-23 A x S-19/05) 42.11\% and 80.00\%) followed by DHLB-23 A x S-19/13) $34.21 \%$ and $70.00 \%$ ) and S-16/769 A $x$ S-19/04) $31.58 \%$ and $66.67 \%$ ) had shown highest standard heterosis over both checks;
Aadishakti and AHB-1200) Fe), respectively. Similar results has been reported by several workers viz., Mungra et al.,) 2014), Patel et $a l .$,$) 2016). With respect to the character plant$ height, not a single hybrid shown significant heterosis in desired direction over better parent. The hybrids viz., S-16/704 A x S19/14) $17.94 \%$ and $19.39 \%$ ) and S-16/704 A x S-19/03) $16.47 \%$ and $18.37 \%$ ) had recorded the highest magnitude of heterosis in desired direction over the standard checks; Aadishakti and AHB-1200) Fe), correspondingly. These results are in accordance with Chittora et al., (2017), Karvar et al., (2017) and Badhe et al., (2018).

Table.1 Analysis of variance for different characters in pearl millet

\begin{tabular}{|l|c|c|c|c|c|c|c|c|c|c|c|}
\hline Source & d.f & DF & DM & NET & PH & EL & EG & TW & GYP & FYP & HI \\
\hline $\begin{array}{l}\text { Replicatio } \\
\text { n }\end{array}$ & 1 & 0.59 & 0.59 & 0.018 & 22.41 & 0.06 & 0.08 & 0.92 & 5.24 & 0.78 & 0.12 \\
\hline Treatment & 53 & $19.93^{* *}$ & $27.03^{* *}$ & $0.23^{* *}$ & $599.3^{* *}$ & $8.08^{* *}$ & $1.30^{* *}$ & $9.61^{* *}$ & $314.2^{* *}$ & $568.4^{* *}$ & $78.18^{* *}$ \\
\hline Parents & 13 & $28.18^{* *}$ & $23.21^{* *}$ & $0.09^{* *}$ & $1514.6^{* *}$ & $14.24^{* *}$ & $1.18^{* *}$ & $3.31^{* *}$ & $73.34^{* *}$ & $604.7^{* *}$ & $76.94^{* *}$ \\
\hline Crosses & 39 & $13.84^{* *}$ & $26.29^{* *}$ & $0.23^{* *}$ & $307.8^{* *}$ & $5.59^{* *}$ & $0.93^{* *}$ & $4.11^{* *}$ & $162.0^{* *}$ & $474.9^{* *}$ & $42.23^{* *}$ \\
\hline $\begin{array}{l}\text { Parents v/s } \\
\text { Crosses }\end{array}$ & 1 & $150.4^{* *}$ & $105.3^{* *}$ & $1.94^{* *}$ & 70.38 & $24.99^{* *}$ & $17.22^{* *}$ & $306.0^{* *}$ & $9381.4^{* *}$ & $3744.0^{* *}$ & $1496.5^{* *}$ \\
\hline Error & 53 & 1.34 & 1.95 & 0.005 & 38.38 & 0.67 & 0.14 & 0.30 & 8.30 & 46.03 & 2.64 \\
\hline
\end{tabular}

* and ** Significant at 5 and 1 per cent level, respectively. Where,

$\begin{array}{llll}\mathrm{DF} & =\text { Days to } 50 \% \text { flowering } & \mathrm{PH}=\text { Plant height }(\mathrm{cm}) & \mathrm{TW}=1000 \text {-seed weight }(\mathrm{g}) \quad \mathrm{HI}= \\ \mathrm{DM}=\text { Days to maturity } & \mathrm{EL}=\text { Earhead length } & \text { GYP }=\text { Grain yield per plant }(\mathrm{g})\end{array}$

NET $=$ No. of effective tillers per plant $\quad$ EG $=$ Earhead girth $\quad$ FY $=$ Fodder yield per plant $(\mathrm{g})$

Table.2 The estimates of heterosis over mid parent (MP), better parent (BP) and standard check (SC) for different characters in pearl millet

\begin{tabular}{|c|l|l|l|l|l|l|l|l|l|}
\hline $\begin{array}{c}\text { Sr. } \\
\text { No. }\end{array}$ & \multicolumn{1}{|c|}{ Crosses } & \multicolumn{3}{|c|}{ Days to 50\% flowering } & \multicolumn{3}{c|}{ Days to maturity } \\
\hline & & M.P.(\%) & \multicolumn{1}{|c|}{ B.P. $(\%)$} & \multicolumn{1}{|c|}{ SC-1 } & \multicolumn{1}{c|}{ SC-2 } & M.P.(\%) & B.P. (\%) & SC-1 & SC-2 \\
\hline $\mathbf{1}$ & 760 A x S-19/03 & $-7.83 * *$ & $-14.52 * *$ & 0.00 & -1.85 & $-4.40 * *$ & $-7.45 * *$ & -2.25 & -1.14 \\
\hline $\mathbf{2}$ & 760 A x S-19/04 & $-7.83 * *$ & $-14.52 * *$ & 0.00 & -1.85 & $-6.01 * *$ & $-9.47 * *$ & $-3.37 *$ & -2.27 \\
\hline $\mathbf{3}$ & 760 A x S-19/05 & $-7.96 * *$ & $-13.33 * *$ & -1.89 & -3.70 & $-6.67 * *$ & $-8.70 * *$ & $-5.62 * *$ & $-4.55 * *$ \\
\hline $\mathbf{4}$ & 760 A x S-19/08 & $-10.53 * *$ & $-16.39 * *$ & -3.77 & $-5.56 *$ & $-8.79 * *$ & $-11.7 * *$ & $-6.74 * *$ & $-5.68 * *$ \\
\hline $\mathbf{5}$ & 760 A x S-19/09 & $-8.26 * *$ & $-10.71 * *$ & $-5.66 *$ & $-7.41 * *$ & $-8.99 * *$ & $-10.0 * *$ & $-8.99 * *$ & $-7.95 * *$ \\
\hline $\mathbf{6}$ & 760 A x S-19/10 & $-4.59 *$ & $-7.14 * *$ & -1.89 & -3.70 & $-5.62 * *$ & $-6.67 * *$ & $-5.62 * *$ & $-4.55 * *$ \\
\hline $\mathbf{7}$ & 760 A x S-19/11 & -0.92 & -3.57 & 1.89 & 0.00 & -1.69 & -2.25 & -2.25 & -1.14 \\
\hline $\mathbf{8}$ & 760 A x S-19/12 & -0.90 & $-5.17 *$ & 3.77 & 1.85 & $-2.79 *$ & $-4.40 * *$ & -2.25 & -1.14 \\
\hline $\mathbf{9}$ & 760 A x S-19/13 & -1.82 & $-5.26 *$ & 1.89 & 0.00 & -2.25 & $-3.33 *$ & -2.25 & -1.14 \\
\hline $\mathbf{1 0}$ & 760 A x S-19/14 & -1.82 & $-5.26 *$ & 1.89 & 0.00 & $-6.15 * *$ & $-7.69 * *$ & $-5.62 * *$ & $-4.55 * *$ \\
\hline
\end{tabular}




\begin{tabular}{|c|c|c|c|c|c|c|c|c|c|}
\hline 11 & 769 A x S-19/03 & -1.75 & $-9.68 * *$ & $5.66 *$ & 3.70 & 1.68 & $-3.19 *$ & 2.25 & $3.41 *$ \\
\hline 12 & 769 A x S-19/04 & $-7.02 * *$ & $-14.52 * *$ & 0.00 & -1.85 & -1.11 & $-6.32 * *$ & 0.00 & 1.14 \\
\hline 13 & 769 A x S-19/05 & $-5.36 * *$ & $-11.67 * *$ & 0.00 & -1.85 & -0.56 & $-4.35 * *$ & -1.12 & 0.00 \\
\hline 14 & 769 A x S-19/08 & -2.65 & $-9.84 * *$ & 3.77 & 1.85 & 0.56 & $-4.26 * *$ & 1.12 & 2.27 \\
\hline 15 & 769 A x S-19/09 & $-7.41 * *$ & $-10.71 * *$ & $-5.66 *$ & $-7.41 * *$ & $-7.43 * *$ & $-10.0 * *$ & $-8.99 * *$ & $-7.95 * *$ \\
\hline 16 & 769 A x S-19/10 & $-7.41 * *$ & $-10.71 * *$ & $-5.66 *$ & $-7.41 * *$ & $-6.29 * *$ & $-8.89 * *$ & $-7.87 * *$ & $-6.82 * *$ \\
\hline 17 & 769 A x S-19/11 & $9.26 * *$ & $5.36 *$ & $11.32 * *$ & $9.26 * *$ & $5.75 * *$ & $3.37 *$ & $3.37 *$ & $4.55 * *$ \\
\hline 18 & 769 A x S-19/12 & $5.45 * *$ & 0.00 & $9.43 * *$ & $7.41 * *$ & $4.55 * *$ & 1.10 & $3.37 *$ & $4.55 * *$ \\
\hline 19 & 769 A x S-19/13 & $6.42 * *$ & 1.75 & $9.43 * *$ & $7.41 * *$ & $5.14 * *$ & 2.22 & $3.37 *$ & $4.55 * *$ \\
\hline 20 & 769 A x S-19/14 & $6.42 * *$ & 1.75 & $9.43 * *$ & $7.41 * *$ & $5.68 * *$ & 2.20 & $4.49 * *$ & $5.68 * *$ \\
\hline 21 & 704 A x S-19/03 & -2.61 & $-9.68 * *$ & $5.66 *$ & 3.70 & 0.55 & $-3.19 *$ & 2.25 & $3.41 *$ \\
\hline 22 & 704 A x S-19/04 & $-4.35 *$ & $-11.29 * *$ & 3.77 & 1.85 & -2.20 & $-6.32 * *$ & 0.00 & 1.14 \\
\hline 23 & 704 A x S-19/05 & $-4.42 *$ & $-10.00 * *$ & 1.89 & 0.00 & -0.56 & $-3.26 *$ & 0.00 & 1.14 \\
\hline 24 & 704 A x S-19/08 & $-5.26 * *$ & $-11.48 * *$ & 1.89 & 0.00 & $-2.76 *$ & $-6.38 * *$ & -1.12 & 0.00 \\
\hline 25 & 704 A x S-19/09 & -2.75 & $-5.36 *$ & 0.00 & -1.85 & -0.56 & -2.22 & -1.12 & 0.00 \\
\hline 26 & 704 A x S-19/10 & $-8.26 * *$ & $-10.71 * *$ & $-5.66 *$ & $-7.41 * *$ & $-3.95 * *$ & $-5.56 * *$ & $-4.49 * *$ & $-3.41 *$ \\
\hline 27 & 704 A x S-19/11 & -0.92 & -3.57 & 1.89 & 0.00 & 2.27 & 1.12 & 1.12 & 2.27 \\
\hline 28 & 704 A x S-19/12 & 0.90 & -3.45 & $5.66 *$ & 3.70 & 2.25 & 0.00 & 2.25 & $3.41 *$ \\
\hline 29 & 704 A x S-19/13 & $5.45 * *$ & 1.75 & $9.43 * *$ & $7.41 * *$ & $5.08 * *$ & $3.33 *$ & $4.49 * *$ & $5.68 * *$ \\
\hline 30 & 704 A x S-19/14 & $5.45 * *$ & 1.75 & $9.43 * *$ & $7.41 * *$ & $5.62 * *$ & $3.30 *$ & $5.62 * *$ & $6.82 * *$ \\
\hline 31 & 23 A x S-19/03 & 0.00 & $-9.68 * *$ & $5.66 *$ & 3.70 & $2.82 *$ & $-3.19 *$ & 2.25 & $3.41 *$ \\
\hline 32 & 23 A x S-19/04 & 0.00 & $-9.68 * *$ & $5.66 *$ & 3.70 & 1.12 & $-5.26 * *$ & 1.12 & 2.27 \\
\hline 33 & 23 A x S-19/05 & $-5.45 * *$ & $-13.33 * *$ & -1.89 & -3.70 & $-4.00 * *$ & $-8.70 * *$ & $-5.62 * *$ & $-4.55 * *$ \\
\hline 34 & 23 A x S-19/08 & -2.70 & $-11.48 * *$ & 1.89 & 0.00 & -1.69 & $-7.45 * *$ & -2.25 & -1.14 \\
\hline 35 & 23 A x S-19/09 & $-7.55 * *$ & $-12.50 * *$ & $-7.55 * *$ & $-9.26 * *$ & $-6.36 * *$ & $-10.00 * *$ & $-8.99 * *$ & $-7.95 * *$ \\
\hline 36 & 23 A x S-19/10 & $-7.55 * *$ & $-12.50 * *$ & $-7.55 * *$ & $-9.26 * *$ & $-5.20 * *$ & $-8.89 * *$ & $-7.87 * *$ & $-6.82 * *$ \\
\hline 37 & 23 A x S-19/11 & 0.00 & $-5.36 *$ & 0.00 & -1.85 & 2.33 & -1.12 & -1.12 & 0.00 \\
\hline 38 & 23 A x S-19/12 & 0.00 & $-6.90 * *$ & 1.89 & 0.00 & 2.30 & -2.20 & 0.00 & 1.14 \\
\hline 39 & 23 A x S-19/13 & 0.93 & $-5.26 *$ & 1.89 & 0.00 & 0.58 & $-3.33 *$ & -2.25 & -1.14 \\
\hline \multirow[t]{4}{*}{40} & 23 A x S-19/14 & 2.80 & -3.51 & 3.77 & 1.85 & $3.45 *$ & -1.10 & 1.12 & 2.27 \\
\hline & $\mathrm{SE}(\mathrm{d})( \pm)$ & 1.005 & 1.160 & 1.160 & 1.160 & 1.209 & 1.396 & 1.396 & 1.396 \\
\hline & CD@95\% & 2.033 & 2.347 & 2.347 & 2.347 & 2.446 & 2.825 & 2.825 & 2.825 \\
\hline & CD @ $99 \%$ & 2.722 & 3.143 & 3.143 & 3.143 & 3.275 & 3.782 & 3.782 & 3.782 \\
\hline
\end{tabular}

\begin{tabular}{|c|c|c|c|c|c|c|c|c|c|}
\hline & & & ber of effec & ve tillers pe & plant & & Plant h & ight (cm) & \\
\hline $\begin{array}{l}\text { Sr. } \\
\text { No. }\end{array}$ & Crosses & M.P.(\%) & B.P. (\%) & SC-1 & SC-2 & M.P.(\%) & B.P. (\%) & SC-1 & SC-2 \\
\hline 1 & 760 A x S-19/03 & $53.33 * *$ & $27.78 * *$ & $21.05 * *$ & $53.33 * *$ & 4.63 & $-13.63 * *$ & 5.22 & 6.94 \\
\hline 2 & 760 A x S-19/04 & $40.00 * *$ & $16.67 * *$ & $10.53 * *$ & $40.00 * *$ & -1.26 & $-17.39 * *$ & -2.68 & -1.09 \\
\hline 3 & 760 A x S-19/05 & $5.48 * *$ & $10.53 * *$ & $10.53 * *$ & $40.00 * *$ & 3.29 & $-13.62 * *$ & 1.87 & 3.54 \\
\hline 4 & 760 A x S-19/08 & $16.13 * *$ & -5.26 & -5.26 & $20.00 * *$ & -1.08 & $-15.65 * *$ & -5.15 & -3.61 \\
\hline 5 & 760 A x S-19/09 & $0.00 * *$ & $15.38 * *$ & $-21.05 * *$ & 0.00 & 4.69 & -2.48 & $-10.37 *$ & $-8.91 *$ \\
\hline 6 & 760 A x S-19/10 & $26.67 * *$ & 5.56 & 0.00 & $26.67 * *$ & 7.34 & -7.15 & 0.87 & 2.52 \\
\hline 7 & 760 A x S-19/11 & $33.33 * *$ & $20.00 * *$ & -5.26 & $20.00 * *$ & 4.16 & $-12.55 * *$ & 2.14 & 3.81 \\
\hline 8 & 760 A x S-19/12 & $21.43 * *$ & 6.25 & $-10.53 * *$ & $13.33 * *$ & 2.50 & $-13.64 * *$ & 0.00 & 1.63 \\
\hline 9 & 760 A x S-19/13 & $40.74 * *$ & $26.67 * *$ & 0.00 & $26.67 * *$ & 6.64 & $-7.91 *$ & 0.47 & 2.11 \\
\hline 10 & 760 A x S-19/14 & $20.00 * *$ & 0.00 & -5.26 & $20.00 * *$ & -5.32 & $-19.13 * *$ & $-9.44 *$ & -7.96 \\
\hline 11 & 769 A x S-19/03 & $33.33 * *$ & $22.22 * *$ & $15.79 * *$ & $46.67 * *$ & $8.85 *$ & $-16.87 * *$ & 1.27 & 2.93 \\
\hline 12 & 769 A x S-19/04 & $51.52 * *$ & $38.89 * *$ & $31.58 * *$ & $66.67 * *$ & 3.01 & $-20.40 * *$ & -6.22 & -4.69 \\
\hline 13 & 769 A x S-19/05 & $23.53 * *$ & $10.53 * *$ & $10.53 * *$ & $40.00 * *$ & $9.11 *$ & $-15.72 * *$ & -0.60 & 1.02 \\
\hline 14 & 769 A x S-19/08 & $17.65 * *$ & 5.26 & 5.26 & $33.33 * *$ & $11.14 * *$ & $-12.68 * *$ & -1.81 & -0.20 \\
\hline
\end{tabular}




\begin{tabular}{|c|c|c|c|c|c|c|c|c|c|}
\hline 15 & 769 A x S-19/09 & 0.00 & -6.67 & $-26.32 * *$ & -6.67 & $9.64 *$ & -6.85 & $-14.39 * *$ & $-12.99 * *$ \\
\hline 16 & 769 A x S-19/10 & $9.09 *$ & 0.00 & -5.26 & $20.00 * *$ & $15.91 * *$ & $-7.76 *$ & 0.20 & 1.84 \\
\hline 17 & 769 A x S-19/11 & $26.67 * *$ & $26.67 * *$ & 0.00 & $26.67 * *$ & $23.11 * *$ & -4.58 & $11.45 * *$ & $13.27 * *$ \\
\hline 18 & 769 A x S-19/12 & 3.23 & 0.00 & $-15.79 * *$ & 6.67 & $25.28 * *$ & -2.60 & $12.78 * *$ & $14.63 * *$ \\
\hline 19 & 769 A x S-19/13 & $20.00 * *$ & $20.00 * *$ & -5.26 & $20.00 * *$ & $27.03 * *$ & 0.92 & $10.11 *$ & $11.90 * *$ \\
\hline 20 & 769 A x S-19/14 & $27.27 * *$ & $16.67 * *$ & $10.53 * *$ & $40.00 * *$ & $30.19 * *$ & 2.45 & $14.73 * *$ & $16.60 * *$ \\
\hline 21 & 704 A x S-19/03 & $11.11 * *$ & $11.11 * *$ & 5.26 & $33.33 * *$ & $10.83 * *$ & -4.40 & $16.47 * *$ & $18.37 * *$ \\
\hline 22 & 704 A x S-19/04 & 5.56 & 5.56 & 0.00 & $26.67 * *$ & 6.36 & -6.93 & $9.64 *$ & $11.43 * *$ \\
\hline 23 & 704 A x S-19/05 & $29.73 * *$ & $26.32 * *$ & $26.32 * *$ & $60.00 * *$ & 2.79 & $-10.10 * *$ & 6.02 & 7.76 \\
\hline 24 & 704 A x S-19/08 & $-18.92 * *$ & $-21.05 * *$ & $-21.05 * *$ & 0.00 & $10.20 * *$ & -1.61 & $10.64 *$ & $12.45 * *$ \\
\hline 25 & 704 A x S-19/09 & $-29.03 * *$ & $-38.89 * *$ & $-42.11 * *$ & $-26.67 * *$ & $10.21 *$ & 8.08 & -0.67 & 0.95 \\
\hline 26 & 704 A x S-19/10 & 5.56 & 5.56 & 0.00 & $26.67 * *$ & $8.87 *$ & -1.29 & 7.23 & $8.98 *$ \\
\hline 27 & 704 A x S-19/11 & $15.15 * *$ & 5.56 & 0.00 & $26.67 * *$ & $9.62 * *$ & -3.72 & $12.45 * *$ & $14.29 * *$ \\
\hline 28 & 704 A x S-19/12 & $35.29 * *$ & $27.78 * *$ & $21.05 * *$ & $53.33 *$ & $10.49 * *$ & -2.60 & $12.78 * *$ & $14.63 * *$ \\
\hline 29 & 704 A x S-19/13 & $27.27 * *$ & $16.67 * *$ & $10.53 * *$ & $40.00 * *$ & $14.58 * *$ & 3.68 & $13.12 * *$ & $14.97 * *$ \\
\hline 30 & 704 A x S-19/14 & $-27.78 * *$ & $-27.78 * *$ & $-31.58 * *$ & $-13.33 * *$ & $17.74 * *$ & 5.32 & $17.94 * *$ & $19.86 * *$ \\
\hline 31 & 23 A x S-19/03 & $17.65 * *$ & $11.11 * *$ & 5.26 & $33.33 * *$ & $15.91 * *$ & -4.95 & $15.80 * *$ & $17.69 * *$ \\
\hline 32 & $23 \mathrm{~A} \times \mathrm{S}-19 / 04$ & $35.29 * *$ & $27.78 * *$ & $21.05 * *$ & $53.33 * *$ & $20.00 * *$ & -0.28 & $17.47 * *$ & $19.39 * *$ \\
\hline 33 & $23 \mathrm{~A} \times \mathrm{S}-19 / 05$ & $54.29 * *$ & $42.11 * *$ & $42.11 * *$ & $80.00 * *$ & $11.72 * *$ & $-7.21 *$ & $9.44 *$ & $11.22 *$ \\
\hline 34 & 23 A x S-19/08 & $20.00 * *$ & $10.53 * *$ & $10.53 * *$ & $40.00 * *$ & $8.54 *$ & $-8.10 *$ & 3.35 & 5.03 \\
\hline 35 & 23 A x S-19/09 & 3.45 & -6.25 & $-21.05 * *$ & 0.00 & $16.23 * *$ & 7.43 & -1.27 & 0.34 \\
\hline 36 & 23 A x S-19/10 & $29.41 * *$ & $22.22 * *$ & $15.79 * *$ & $46.67 * *$ & 4.81 & $-9.98 *$ & -2.21 & -0.61 \\
\hline 37 & 23 A x S-19/11 & $29.03 * *$ & $25.00 * *$ & 5.26 & $33.33 * *$ & 3.09 & $-19.20 * *$ & -5.62 & -4.08 \\
\hline 38 & 23 A x S-19/12 & 6.25 & 6.25 & $-10.53 * *$ & $13.33 * *$ & -1.90 & $-17.92 * *$ & -4.95 & -3.40 \\
\hline 39 & $23 \mathrm{~A} \times \mathrm{S}-19 / 13$ & $64.52 * *$ & $59.37 * *$ & $34.21 * *$ & $70.00 * *$ & 5.76 & $-9.33 *$ & -1.07 & 0.54 \\
\hline \multirow[t]{4}{*}{40} & 23 A x S-19/14 & $29.41 * *$ & $22.22 * *$ & $15.79 * *$ & $6.67 * *$ & 5.14 & $-10.82 * *$ & -0.13 & 1.50 \\
\hline & SE (d) $( \pm)$ & 0.063 & 0.072 & 0.072 & 0.072 & 5.365 & 6.195 & 6.195 & 6.195 \\
\hline & CD@95\% & 0.127 & 0.147 & 0.147 & 0.147 & 10.852 & 12.531 & 12.531 & 12.531 \\
\hline & CD@99\% & 0.171 & 0.197 & 0.197 & 0.197 & 14.528 & 16.776 & 16.776 & 16.776 \\
\hline & & \multicolumn{4}{|c|}{ Earhead length $(\mathrm{cm})$} & \multicolumn{4}{|c|}{ Earhead girth $(\mathrm{cm})$} \\
\hline $\begin{array}{l}\text { Sr. } \\
\text { No. }\end{array}$ & Crosses & M.P.(\%) & B.P. (\%) & SC-1 & SC-2 & M.P.(\%) & B.P. (\%) & SC-1 & SC-2 \\
\hline 1 & 760 A x S-19/03 & 5.09 & $-7.31 *$ & 4.47 & 5.46 & 5.07 & -1.44 & -3.05 & $-11.58 * *$ \\
\hline 2 & 760 A x S-19/04 & $8.80 *$ & -1.55 & 4.71 & 5.70 & $8.44 *$ & -0.05 & -1.69 & $-10.34 * *$ \\
\hline 3 & 760 A x S-19/05 & 2.13 & $-10.00 * *$ & 1.65 & 2.61 & $9.31 *$ & 0.57 & -1.08 & $-9.78 * *$ \\
\hline 4 & 760 A x S-19/08 & -5.80 & $-19.30 * *$ & -2.59 & -1.66 & $9.88 * *$ & 4.42 & 2.71 & -6.33 \\
\hline 5 & 760 A x S-19/09 & $10.31 * *$ & 4.39 & 0.71 & 1.66 & 0.81 & -2.30 & 2.41 & -6.60 \\
\hline 6 & 760 A x S-19/10 & $15.99 * *$ & $8.29 *$ & 7.53 & $8.55 *$ & 6.30 & 4.58 & 2.86 & -6.19 \\
\hline 7 & 760 A x S-19/11 & $14.76 * *$ & 1.04 & $14.35 * *$ & $15.44 * *$ & $9.48 * *$ & $9.28 *$ & 7.88 & -1.61 \\
\hline 8 & 760 A x S-19/12 & 3.11 & $-10.93 * *$ & 5.41 & 6.41 & $7.77 *$ & 4.45 & $9.49 *$ & -0.15 \\
\hline 9 & 760 A x S-19/13 & 6.60 & $-7.44 *$ & $8.24 *$ & $9.26 *$ & 6.48 & 5.44 & 5.78 & -3.52 \\
\hline 10 & 760 A x S-19/14 & $8.57 *$ & -3.80 & 7.29 & $8.31 *$ & 4.35 & 2.36 & 4.66 & -4.55 \\
\hline 11 & 769 A x S-19/03 & $10.15 * *$ & 0.84 & $13.65 * *$ & $14.73 * *$ & -1.10 & -2.01 & $-13.98 * *$ & $-21.55 * *$ \\
\hline 12 & 769 A x S-19/04 & 4.24 & -1.99 & 4.24 & 5.23 & 6.59 & 3.66 & $-9.00 *$ & $-17.01 * *$ \\
\hline 13 & 769 A x S-19/05 & $9.11 * *$ & -0.21 & $2.71 * *$ & $13.78 * *$ & $12.08 * *$ & 8.79 & -4.50 & $-12.9 * *$ \\
\hline 14 & 769 A x S-19/08 & $9.33 * *$ & -2.92 & $17.18 * *$ & $18.29 * *$ & $17.05 * *$ & $16.52 * *$ & 3.22 & -5.87 \\
\hline 15 & 769 A x S-19/09 & $15.35 * *$ & $13.66 * *$ & $9.65 *$ & $10.69 * *$ & $9.02 *$ & 0.15 & 4.98 & -4.25 \\
\hline 16 & 769 A x S-19/10 & $20.98 * *$ & $17.54 * *$ & $16.71 * *$ & $17.81 * *$ & $12.65 * *$ & 8.28 & 3.05 & -6.01 \\
\hline 17 & 769 A x S-19/11 & $16.50 * *$ & 6.44 & $20.47 * *$ & $21.62 * *$ & $19.66 * *$ & $13.03 * *$ & $11.58 * *$ & 1.76 \\
\hline 18 & 769 A x S-19/12 & $15.21 * *$ & 3.18 & $22.12 * *$ & $23.28 * *$ & $13.86 * *$ & 4.60 & $9.65 *$ & 0.00 \\
\hline
\end{tabular}




\begin{tabular}{|c|c|c|c|c|c|c|c|c|c|}
\hline 19 & 769 A x S-19/13 & $16.20 * *$ & 4.63 & $22.35 * *$ & $23.52 * *$ & $18.29 * *$ & $10.90 * *$ & $11.25 * *$ & 1.47 \\
\hline 20 & 769 A x S-19/14 & $17.89 * *$ & $8.44 *$ & $20.94 * *$ & $22.09 * *$ & $16.07 * *$ & $7.86 *$ & $10.29 *$ & 0.59 \\
\hline 21 & 704 A x S-19/03 & $9.30 * *$ & 0.63 & $13.41 * *$ & $14.49 * *$ & $9.70 *$ & 3.69 & 0.35 & $-8.47 *$ \\
\hline 22 & 704 A x S-19/04 & $12.05 * *$ & 5.97 & $12.71 * *$ & $13.78 * *$ & $10.35 * *$ & 2.47 & -0.82 & $-9.55 *$ \\
\hline 23 & 704 A x S-19/05 & $7.81 *$ & -0.83 & $12.00 * *$ & $13.06 * *$ & 5.38 & -2.33 & -5.47 & $-13.78 * *$ \\
\hline 24 & 704 A x S-19/08 & $7.64 *$ & -3.90 & $16.00 * *$ & $17.10 * *$ & $15.52 * *$ & $10.63 *$ & 7.07 & -2.35 \\
\hline 25 & 704 A x S-19/09 & $18.57 * *$ & $17.56 * *$ & $13.41 * *$ & $14.49 * *$ & 6.70 & 2.61 & 7.56 & -1.91 \\
\hline 26 & 704 A x S-19/10 & $14.18 * *$ & $11.61 * *$ & $10.82 * *$ & $11.88 * *$ & $10.22 * *$ & $9.30 *$ & 5.79 & -3.52 \\
\hline 27 & 704 A x S-19/11 & 5.88 & -2.70 & $10.12 *$ & $11.16 * *$ & $15.95 * *$ & $14.82 * *$ & $13.34 * *$ & 3.37 \\
\hline 28 & 704 A x S-19/12 & $11.26 * *$ & 0.20 & $18.59 * *$ & $19.71 * *$ & $16.43 * *$ & $11.96 * *$ & $17.36 * *$ & 7.04 \\
\hline 29 & 704 A x S-19/13 & $15.11 * *$ & 4.23 & $21.88 * *$ & $23.04 * *$ & $12.72 * *$ & $10.74 * *$ & $11.09 * *$ & 1.32 \\
\hline 30 & 704 A x S-19/14 & $17.90 * *$ & $9.07 *$ & $21.65 * *$ & $22.80 * *$ & $15.67 * *$ & $12.58 * *$ & $15.11 * *$ & 4.99 \\
\hline 31 & 23 A x S-19/03 & $15.88 * *$ & -0.21 & $12.47 * *$ & $13.54 * *$ & $12.03 * *$ & 3.82 & 4.82 & -4.40 \\
\hline 32 & $23 \mathrm{~A} \times \mathrm{S}-19 / 04$ & $14.54 * *$ & 1.11 & 7.53 & $8.55 *$ & $13.99 * *$ & 3.82 & 4.82 & -4.40 \\
\hline 33 & $23 \mathrm{~A} \times \mathrm{S}-19 / 05$ & 2.66 & $-11.67 * *$ & -0.24 & 0.71 & 0.39 & $-8.72 *$ & -7.84 & $-15.95 * *$ \\
\hline 34 & $23 \mathrm{~A} \times \mathrm{S}-19 / 08$ & $7.57 *$ & $-9.94 * *$ & $8.71 *$ & $9.74 *$ & $13.32 * *$ & 6.37 & 7.40 & -2.05 \\
\hline 35 & 23 A x S-19/09 & $12.43 * *$ & 3.66 & 0.00 & 0.95 & $7.34 *$ & 5.37 & $10.45 *$ & 0.73 \\
\hline 36 & $23 \mathrm{~A} \times \mathrm{S}-19 / 10$ & 3.39 & -5.92 & -6.59 & -5.70 & -2.37 & -5.17 & -4.25 & $-12.68 * *$ \\
\hline 37 & $23 \mathrm{~A} \times \mathrm{S}-19 / 11$ & -3.26 & $-16.84 * *$ & -5.88 & -4.99 & -0.51 & -1.62 & -0.67 & $-9.41 *$ \\
\hline 38 & $23 \mathrm{~A} \times \mathrm{S}-19 / 12$ & 1.06 & $-14.71 * *$ & 0.94 & 1.90 & $8.44 *$ & 6.44 & $11.58^{* *}$ & 1.76 \\
\hline 39 & 23 A x S-19/13 & $10.08 * *$ & -6.64 & $9.18 *$ & $10.21 *$ & $9.58 * *$ & $9.24 *$ & $10.29 *$ & 0.59 \\
\hline 40 & $23 \mathrm{~A} \times \mathrm{S}-19 / 14$ & 6.10 & $-8.23 *$ & 2.35 & 3.33 & $8.07 *$ & 7.39 & $9.81 *$ & 0.15 \\
\hline & $\mathrm{SE}(\mathrm{d})( \pm)$ & 0.713 & 0.823 & 0.823 & 0.823 & 0.332 & 0.384 & 0.384 & 0.384 \\
\hline & CD@95\% & 1.442 & 1.665 & 1.665 & 1.665 & 0.672 & 0.770 & 0.777 & 0.777 \\
\hline & CD@99\% & 1.931 & 2.229 & 2.229 & 2.229 & 0.900 & 1.040 & 1.040 & 1.040 \\
\hline & & \multicolumn{4}{|c|}{1000 seed weight $(\mathrm{g})$} & \multicolumn{4}{|c|}{ Grain yield per plant (g) } \\
\hline $\begin{array}{l}\text { Sr. } \\
\text { No. }\end{array}$ & Crosses & M.P.(\%) & B.P. (\%) & SC-1 & SC-2 & M.P.(\%) & B.P. (\%) & SC-1 & SC-2 \\
\hline 1 & 760 A x S-19/03 & $35.98 * *$ & $29.06 * *$ & $17.93 * *$ & $20.89 * *$ & $61.09 * *$ & $46.69 * *$ & -6.74 & 9.93 \\
\hline 2 & 760 A x S-19/04 & $36.89 * *$ & $33.60 * *$ & $15.17 * *$ & $18.06 * *$ & $69.66 * *$ & $67.98 * *$ & -10.53 & 5.46 \\
\hline 3 & 760 A x S-19/05 & $45.73 * *$ & $39.92 * *$ & $14.83 * *$ & $17.71 * *$ & $62.29 * *$ & $39.31 * *$ & 1.47 & $19.60 *$ \\
\hline 4 & 760 A x S-19/08 & $46.98 * *$ & $32.77 * *$ & $8.97 *$ & $11.70 * *$ & $78.15 * *$ & $51.21 * *$ & $-21.05 * *$ & -6.95 \\
\hline 5 & 760 A x S-19/09 & $37.16 * *$ & $25.63 * *$ & 3.10 & 5.69 & $37.91 * *$ & 17.34 & $-38.74 * *$ & $-27.79 * *$ \\
\hline 6 & 760 A x S-19/10 & $18.90 * *$ & $11.85 * *$ & 4.14 & 6.75 & $36.05 * *$ & 11.28 & -8.63 & 7.69 \\
\hline 7 & 760 A x S-19/11 & $29.08 * *$ & $22.73 * *$ & $11.72 * *$ & $14.53 * *$ & $82.83 * *$ & $81.03 * *$ & -3.58 & 13.65 \\
\hline 8 & 760 A x S-19/12 & $25.34 * *$ & $16.13 * *$ & $11.72 * *$ & $14.53 * *$ & $58.59 * *$ & $53.79 * *$ & $-14.53 *$ & 0.74 \\
\hline 9 & 760 A x S-19/13 & $26.40 * *$ & $24.54 * *$ & 2.21 & 4.77 & $62.63 * *$ & $54.44 * *$ & $-19.37 * *$ & -4.96 \\
\hline 10 & 760 A x S-19/14 & $47.68 * *$ & $44.42 * *$ & $24.00 * *$ & $27.11 * *$ & $71.48 * *$ & $64.21 * *$ & -6.32 & 10.42 \\
\hline 11 & 769 A x S-19/03 & $20.38 * *$ & $18.57 * *$ & $8.34 *$ & $11.06 * *$ & $64.89 * *$ & $38.41 * *$ & -12.00 & 3.72 \\
\hline 12 & 769 A x S-19/04 & $20.51 * *$ & $18.87 * *$ & 5.34 & $7.99 *$ & $88.21 * *$ & $70.36 * *$ & -9.26 & 6.95 \\
\hline 13 & 769 A x S-19/05 & $34.50 * *$ & $24.55 * *$ & $10.38 * *$ & $13.15 * *$ & $67.70 * *$ & $33.53 * *$ & -2.74 & 14.64 \\
\hline 14 & 769 A x S-19/08 & $46.06 * *$ & $27.59 * *$ & $13.07 * *$ & $15.91 * *$ & $166.14 * *$ & $145.37 * *$ & 5.89 & $24.81 * *$ \\
\hline 15 & 769 A x S-19/09 & $46.86 * *$ & $30.00 * *$ & $15.21 * *$ & $18.10 * *$ & $107.39 * *$ & $91.71 * *$ & $-17.26 * *$ & -2.48 \\
\hline 16 & 769 A x S-19/10 & $24.40 * *$ & $21.41 * *$ & $13.03 * *$ & $15.87 * *$ & $84.20 * *$ & $40.51 * *$ & $15.37 *$ & $35.98 * *$ \\
\hline 17 & 769 A x S-19/11 & $42.80 * *$ & $40.91 * *$ & $28.28 * *$ & $31.50 * *$ & $172.05 * *$ & $146.25^{* *}$ & $31.16 * *$ & $54.59 * *$ \\
\hline 18 & 769 A x S-19/12 & $31.27 * *$ & $26.09 * *$ & $21.31 * *$ & $24.35 * *$ & $135.39 * *$ & $109.09 * *$ & $16.21 *$ & $36.97 * *$ \\
\hline 19 & 769 A x S-19/13 & $39.47 * *$ & $32.41 * *$ & $17.34 * *$ & $20.29 * *$ & $200.47 * *$ & $188.34 * *$ & $35.37 * *$ & $59.55 * *$ \\
\hline 20 & 769 A x S-19/14 & $35.73 * *$ & $33.62 * *$ & $18.41 * *$ & $21.39 * *$ & $131.51 * *$ & $103.32 * *$ & $16.00 *$ & $36.72 * *$ \\
\hline 21 & 704 A x S-19/03 & $38.15 * *$ & $32.68 * *$ & $21.24 * *$ & $24.28 * *$ & $44.79 * *$ & $44.55 * *$ & -7.79 & 8.68 \\
\hline 22 & 704 A x S-19/04 & $22.31 * *$ & $20.84 * *$ & 4.17 & 6.79 & $45.68 * *$ & $33.66 * *$ & $-14.74 *$ & 0.50 \\
\hline
\end{tabular}




\begin{tabular}{|c|c|c|c|c|c|c|c|c|c|}
\hline 23 & 704 A x S-19/05 & 6.91 & 1.43 & $-14.66 * *$ & $-12.51 * *$ & -2.00 & -8.09 & $-33.05 * *$ & $-21.09 * *$ \\
\hline 24 & 704 A x S-19/08 & $26.42 * *$ & $12.95 * *$ & -4.97 & -2.58 & $66.81 * *$ & $31.02 * *$ & $-16.42 *$ & -1.49 \\
\hline 25 & 704 A x S-19/09 & $43.35 * *$ & $29.84 * *$ & $9.24 *$ & $11.98 * *$ & $24.53 *$ & -1.98 & $-37.47 * *$ & $-26.30 * *$ \\
\hline 26 & 704 A x S-19/10 & $18.87 * *$ & $13.15 * *$ & 5.34 & $7.99 *$ & $40.26 * *$ & $24.62 * *$ & 2.32 & $20.60 * *$ \\
\hline 27 & 704 A x S-19/11 & $44.06 * *$ & $38.60 * *$ & $26.17 * *$ & $29.34 * *$ & $101.08 * *$ & $84.49 * *$ & $17.68 * *$ & $38.71 * *$ \\
\hline 28 & 704 A x S-19/12 & $36.67 * *$ & $28.10 * *$ & $23.24 * *$ & $26.33 * *$ & $147.97 * *$ & $132.01 * *$ & $48.00 * *$ & $74.44 * *$ \\
\hline 29 & 704 A x S-19/13 & $41.56 * *$ & $37.79 * *$ & $15.93 * *$ & $18.84 * *$ & $133.08 * *$ & $102.31 * *$ & $29.05 * *$ & $52.11 * *$ \\
\hline 30 & 704 A x S-19/14 & $42.11 * *$ & $40.68 * *$ & $20.79 * *$ & $23.82 * *$ & $65.16 * *$ & $56.44 * *$ & -0.21 & $17.62 *$ \\
\hline 31 & 23 A x S-19/03 & $19.60 * *$ & $13.96 * *$ & 4.14 & 6.75 & $87.09 * *$ & $77.48 * *$ & $12.84 *$ & $33.00 * *$ \\
\hline 32 & 23 A x S-19/04 & $19.92 * *$ & $17.52 * *$ & 1.31 & 3.85 & $121.37 * *$ & $114.02 * *$ & $22.11 * *$ & $43.92 * *$ \\
\hline 33 & 23 A x S-19/05 & $10.72 *$ & 5.87 & $-12.38 * *$ & $-10.18 *$ & $69.21 * *$ & $50.87 * *$ & 9.89 & $29.53 * *$ \\
\hline 34 & 23 A x S-19/08 & $38.66 * *$ & $24.79 * *$ & 3.28 & 5.87 & $112.16^{* *}$ & $73.80 * *$ & -0.84 & $16.87^{*}$ \\
\hline 35 & 23 A x S-19/09 & $44.89 * *$ & $32.21 * *$ & $9.41 *$ & $12.16 * *$ & $90.56 * *$ & $56.46 * *$ & -10.74 & 5.21 \\
\hline 36 & 23 A x S-19/10 & $17.02 * *$ & $10.52 *$ & 2.90 & 5.48 & $56.13 * *$ & $32.31 * *$ & 8.63 & $28.04 * *$ \\
\hline 37 & 23 A x S-19/11 & $12.70 * *$ & 7.58 & -2.07 & 0.39 & $91.98 * *$ & $85.61 * *$ & 5.89 & $24.81 * *$ \\
\hline 38 & 23 A x S-19/12 & $28.71 * *$ & $19.71 * *$ & $15.17 * *$ & $18.06 * *$ & $90.28 * *$ & $87.82 * *$ & 7.16 & $26.30 * *$ \\
\hline 39 & 23 A x S-19/13 & $43.01 * *$ & $40.33 * *$ & $16.14 * *$ & $19.05 * *$ & $104.05^{* *}$ & $85.98 * *$ & 6.11 & $25.06 * *$ \\
\hline \multirow[t]{4}{*}{40} & 23 A x S-19/14 & $14.60 * *$ & $12.53 * *$ & -3.38 & -0.95 & $89.30 * *$ & $89.30 * *$ & 8.00 & $27.30 * *$ \\
\hline & SE (d) ( $( \pm)$ & 0.474 & 0.548 & 0.548 & 0.548 & 2.581 & 2.980 & 2.980 & 2.980 \\
\hline & CD@ $95 \%$ & 0.959 & 1.108 & 1.108 & 1.108 & 5.221 & 6.029 & 6.029 & 6.029 \\
\hline & CD@ $99 \%$ & 1.285 & 1.483 & 1.483 & 1.483 & 6.990 & 8.071 & 8.071 & 8.071 \\
\hline & & \multicolumn{4}{|c|}{ Fodder yield per plant (g) } & \multicolumn{4}{|c|}{ Harvest index (\%) } \\
\hline $\begin{array}{l}\text { Sr. } \\
\text { No. }\end{array}$ & Crosses & M.P.(\%) & B.P. (\%) & SC-1 & SC-2 & M.P.(\%) & B.P. (\%) & SC-1 & SC-2 \\
\hline 1 & 760 A x S-19/03 & $22.83 *$ & -7.61 & -5.56 & $48.60 * *$ & $15.05 * *$ & -1.69 & -0.85 & $-17.21 * *$ \\
\hline 2 & 760 A x S-19/04 & $31.84 * *$ & 4.83 & -8.44 & $44.06 * *$ & $16.83 * *$ & -0.83 & 0.01 & $-16.48 * *$ \\
\hline 3 & 760 A x S-19/05 & 12.37 & -14.12 & $-16.22 *$ & $31.82 *$ & $23.73 * *$ & $11.98 *$ & $12.94 * *$ & -5.69 \\
\hline 4 & 760 A x S-19/08 & -19.47 & -23.24 & $-56.33 * *$ & $-31.29 *$ & $63.71 * *$ & $40.98 * *$ & $42.18 * *$ & $18.73 * *$ \\
\hline 5 & 760 A x S-19/09 & -21.35 & -23.20 & $-58.44 * *$ & $-34.62 * *$ & $42.87 * *$ & $25.41 * *$ & $26.48 * *$ & 5.62 \\
\hline 6 & 760 A x S-19/10 & 15.10 & 12.84 & $-39.44 * *$ & -4.72 & $11.79 * *$ & -0.39 & $28.46 * *$ & 7.27 \\
\hline 7 & 760 A x S-19/11 & $31.42 *$ & 28.45 & $-33.78 * *$ & 4.20 & $22.49 * *$ & $20.19 * *$ & $25.94 * *$ & 5.17 \\
\hline 8 & 760 A x S-19/12 & 6.84 & 0.19 & $-41.00 * *$ & -7.17 & $28.01 * *$ & $24.92 * *$ & $25.99 * *$ & 5.21 \\
\hline 9 & 760 A x S-19/13 & 6.33 & 4.13 & $-44.00 * *$ & -11.89 & $30.27 * *$ & $23.86 * *$ & $24.92 * *$ & 4.31 \\
\hline 10 & 760 A x S-19/14 & -14.23 & -16.67 & $-54.44 * *$ & $-28.32 *$ & $47.52 * *$ & $45.75 * *$ & $50.60 * *$ & $25.76 * *$ \\
\hline 11 & 769 A x S-19/03 & $27.37 * *$ & -12.50 & -10.56 & $40.73 * *$ & $10.15 *$ & -8.51 & -1.07 & $-17.39 * *$ \\
\hline 12 & 769 A x S-19/04 & $27.43 *$ & -8.40 & $-20.00 *$ & $25.87 *$ & $21.66 * *$ & 0.41 & 8.58 & $-9.33 *$ \\
\hline 13 & 769 A x S-19/05 & $21.11 *$ & $-15.72 *$ & $-17.78 *$ & $29.37 *$ & $17.11 * *$ & 2.80 & $11.16 *$ & -7.18 \\
\hline 14 & 769 A x S-19/08 & $66.12 * *$ & $38.87 * *$ & $-21.00 * *$ & $24.30 *$ & $33.43 * *$ & $11.66 *$ & $20.74 * *$ & 0.82 \\
\hline 15 & 769 A x S-19/09 & 21.54 & 3.70 & $-43.89 * *$ & -11.71 & $37.55 * *$ & $17.24 * *$ & $26.77 * *$ & 5.86 \\
\hline 16 & 769 A x S-19/10 & $45.10 * *$ & 24.22 & $-33.33 * *$ & 4.90 & $16.43 * *$ & 7.02 & $38.03 * *$ & $15.26 * *$ \\
\hline 17 & 769 A x S-19/11 & $108.64 * *$ & $85.33 * *$ & -8.78 & $43.53 * *$ & $17.54 * *$ & $15.72 * *$ & $25.13 * *$ & 4.49 \\
\hline 18 & 769 A x S-19/12 & $70.94 * *$ & $40.94 * *$ & $-17.00 *$ & $30.59 *$ & $22.12 * *$ & $15.27 * *$ & $24.64 * *$ & 4.08 \\
\hline 19 & 769 A x S-19/13 & $57.00 * *$ & $34.30 *$ & $-27.78 * *$ & 13.64 & $44.60 * *$ & $33.09 * *$ & $43.92 * *$ & $20.18 * *$ \\
\hline 20 & 769 A x S-19/14 & $101.67 * *$ & $71.34 * *$ & -6.33 & $47.38 * *$ & $8.16 *$ & 5.75 & $14.35 * *$ & -4.51 \\
\hline 21 & 704 A x S-19/03 & $23.46 * *$ & -6.20 & -4.11 & $50.87 * *$ & 6.47 & $-12.89 * *$ & -2.13 & $-18.27 * *$ \\
\hline 22 & 704 A x S-19/04 & 2.53 & $-17.56 *$ & $-28.00 * *$ & 13.29 & $21.75 * *$ & -1.00 & $11.23 *$ & -7.12 \\
\hline 23 & 704 A x S-19/05 & 1.47 & $-21.64 * *$ & $-23.56 * *$ & 20.28 & -5.61 & $-18.49 * *$ & -8.42 & $-23.53 * *$ \\
\hline 24 & 704 A x S-19/08 & 14.14 & 10.35 & $-37.22 * *$ & -1.22 & $29.06 * *$ & 6.36 & $19.51 * *$ & -0.21 \\
\hline 25 & 704 A x S-19/09 & 3.83 & 2.87 & $-44.33 * *$ & -12.41 & $14.31 * *$ & -4.08 & 7.77 & $-10.00 *$ \\
\hline 26 & 704 A x S-19/10 & 21.75 & 21.12 & $-35.00 * *$ & 2.27 & $8.80 *$ & 1.80 & $31.28 * *$ & $9.63 *$ \\
\hline
\end{tabular}




\begin{tabular}{|c|c|c|c|c|c|c|c|c|c|}
\hline 27 & 704 A x S-19/11 & $83.93 * *$ & $77.20 * *$ & -5.89 & $48.08 * *$ & 5.91 & 2.34 & $14.99 * *$ & -3.98 \\
\hline 28 & 704 A x S-19/12 & $84.52 * *$ & $75.47 * *$ & 3.33 & $62.59 * *$ & $19.55 * *$ & $10.84 *$ & $24.54 * *$ & 4.00 \\
\hline 29 & 704 A x S-19/13 & $55.93 * *$ & $54.96 * *$ & $-16.67 *$ & $31.12 *$ & $28.02 * *$ & $15.81 * *$ & $30.13 * *$ & $8.66 *$ \\
\hline 30 & 704 A x S-19/14 & $36.91 * *$ & $34.96 *$ & $-26.22 * *$ & 16.08 & $11.93 * *$ & 7.43 & $20.71 * *$ & 0.80 \\
\hline 31 & 23 A x S-19/03 & $26.36 * *$ & -5.43 & -3.33 & $52.10 * *$ & $23.00 * *$ & 2.28 & $10.29 *$ & -7.90 \\
\hline 32 & 23 A x S-19/04 & $51.25 * *$ & $19.59 *$ & 4.44 & $64.34 * *$ & $23.95 * *$ & 2.42 & $10.43 *$ & -7.78 \\
\hline 33 & 23 A x S-19/05 & $48.76 * *$ & 13.10 & 10.33 & $73.60 * *$ & 5.31 & -7.45 & -0.20 & $-16.66 * *$ \\
\hline 34 & 23 A x S-19/08 & $41.80 * *$ & $34.18 *$ & $-23.67 * *$ & 20.10 & $30.32 * *$ & $9.18 *$ & $17.73 * *$ & -1.69 \\
\hline 35 & 23 A x S-19/09 & 13.56 & 10.06 & $-40.44 * *$ & -6.29 & $39.03 * *$ & $18.64 * *$ & $27.93 * *$ & 6.83 \\
\hline 36 & 23 A x S-19/10 & $33.19 *$ & $29.61 *$ & $-30.44 * *$ & 9.44 & $10.65 * *$ & 1.58 & $31.01 * *$ & $9.40 *$ \\
\hline 37 & 23 A x S-19/11 & $56.00 * *$ & $53.61 * *$ & $-22.00 * *$ & 22.73 & $13.62 * *$ & $12.02 * *$ & $20.79 * *$ & 0.87 \\
\hline 38 & 23 A x S-19/12 & $62.11 * *$ & $50.94 * *$ & -11.11 & $39.86 * *$ & $10.38 *$ & 4.32 & $12.49 *$ & -6.07 \\
\hline 39 & 23 A x S-19/13 & $36.66 * *$ & $32.85 *$ & $-28.56 * *$ & 12.41 & $28.16 * *$ & $18.12 * *$ & $27.36 * *$ & 6.36 \\
\hline \multirow[t]{4}{*}{40} & 23 A x S-19/14 & $64.17 * *$ & $58.33 * *$ & -13.44 & $36.19 * *$ & $8.81 *$ & 6.54 & $14.87 * *$ & -4.07 \\
\hline & $\mathrm{SE}(\mathrm{d})( \pm)$ & 5.875 & 6.784 & 6.784 & 6.784 & 1.407 & 1.625 & 1.625 & 1.625 \\
\hline & CD@95\% & 11.884 & 13.723 & 13.723 & 13.723 & 2.847 & 3.287 & 3.287 & 3.287 \\
\hline & CD@ $99 \%$ & 15.911 & 18.372 & 18.372 & 18.372 & 3.812 & 4.401 & 4.401 & 4.401 \\
\hline
\end{tabular}

* and ** Significant at 5 and 1 per cent level, respectively.

Where, SC-1 = Aadishakti $\quad$ SC-2 = AHB-1200 (Fe)

The cross S-16/769 A x S-19/10 recorded highest significant positive value over mid parent) $20.98 \%$ ), the cross S-16/704 A x S19/09) $17.56 \%$ ) followed by S-16/769 A x S19/10) $17.54 \%$ ) and S-16/769 A x S-19/09) $13.66 \%$ ) manifested highest significant positive heterosis over better parent, while highest significant and positive heterosis over both checks; Aadishakti) 22.35\%) and AHB1200) Fe)) 23.52\%) exhibited by cross $\mathrm{S}$ 16/769 A x S-19/13 followed by S-16/769 A x S-19/12) $22.12 \%$ and $23.28 \%$ ) respectively for the character earhead length. In case of earhead girth, the cross S-16/769 A x S19/11) $19.66 \%$ ) and S-16/769 A x S-19/08) $16.52 \%$ ) registered highest significant positive average heterosis and heterobeltosis respectively. While, cross S-16/704 A x S19/12 exhibited highest significantly positive heterosis over both standard checks; Aadishakti) 17.36\%) and AHB-1200) Fe) 7.04\%) followed by S-16/704 A x S-19/14) $15.11 \%$ and $4.99 \%$ ) respectively. The results are in the agreement with Athoni et al.,) 2016) and Acharya et al.,) 2017).

With regards to the character 1000 seed weight, the cross S-16/760 A x S-19/14 exhibited highest significant positive heterosis over mid parent) $47.68 \%$ ) and better parent) $44.42 \%$ ). Whereas, the cross S-16/769 A x S$19 / 11$ manifested positively significant heterosis over both standard checks; Adishakti) 28.28\%) and AHB-1200) Fe) $31.50 \%$ ) followed by S-16/704 A x S-19/11) $26.17 \%$ and $29.34 \%$ ) and S-16/760 A x S19/14) $24.00 \%$ and $27.11 \%$ )) respectively. Significant positive heterosis for earhead girth in pearl millet has been reported by several workers viz., Bachkar et al.) 2014) and Krishnan et al.) 2017). For major economic character) grain yield per plant), The highest heterosis over mid parent) $200.47 \%$ ) and over better parent) $188.34 \%$ ) was depicted by the cross S-16/769 A x S-19/13. While, the cross, S-16/704 A x S-19/12 depicted highest positively significant heterosis over both standard checks; Aadishakti) $48.00 \%$ ) and AHB-1200) Fe) $74.44 \%$ ) followed by S16/769 A x S-19/13) 35.37\% and 59.55\%) and S-16/769 A x S-19/11) 31.16\% and $54.59 \%$ ) respectively. The similar findings were also in accordance with previous workers Karvar et al.,) 2017), Gawali et al.,) 2017) and Ladumor et al.,) 2018).

In case of fodder yield per plant, The cross, S16/769 A x S-19/11 registered highest 
significant positive heterosis over mid parent) $108.64 \%$ ) and better parent) $85.33 \%$ ). While the cross, DHLB-23 A x S-19/05 exhibited significantly positive heterosis over standard check Aadishakti) 10.33\%) and AHB-1200) Fe)) $73.60 \%$ ) followed by DHLB-23 A x S19/04) 4.44\% and 64.34\%) and S-16/704 A x S-19/12) $3.33 \%$ and $62.59 \%$ ), respectively. For the character harvest index, The cross, S16/760 A x S-19/08) 63.71\%) and S-16/760 A x S-19/14) 45.75\%) had exhibited positively significant heterosis over mid parent and better parent respectively. While, The cross, S-16/760 A x S-19/14 registered significantly positive heterosis over standard check Adishakti) 50.60\%) and AHB-1200) Fe)) $25.76 \%$ ) followed by S-16/769 A x S-19/13) $43.92 \%$ and $20.18 \%$ ) respectively. Similar results has been reported by Karvar et al., (2017) and Krishnan et al., (2017).

In conclusion the highest values of average heterosis) 200.47 per cent), heterobeltosis) 188.34 per cent) and standard heterosis over standard checks; Aadishakti and AHB-1200) Fe) were 48 per cent and 74.44 per cent, respectively for grain yield per plant. Five crosses viz., S-16/704 A x S-19/12, S-16/769 A x S-19/13, S-16/769 A x S-19/11, S-16/704 A x S-19/13 and DHLB-23 A x S-19/04 manifested significant and positive heterosis over standard checks; Aadishakti and AHB1200) Fe). The above mentioned highly heterotic crosses also occupied top ranks in per se performance for grain yield per plant. Above mentioned top crosses could be checked for their stable performance for multi location trials over environment.

\section{References}

Acharya, Z. R., Khanapara, M.D., Chaudhari V.B. and Dobaria, J. D. (2017). Exploitation of heterosis in pearl millet (Pennisetum glaucum (L) R. Br.) for yield and its component traits by using male sterile line. International Journal of Current Microbiology and Applied Sciences, 6(12): 750-759.

Anonymous. (2018). Area, production and productivity of pearl millet. Research review committee meeting held on 15 $16^{\text {th }}$ Jan. 2017. M.P.K.V Rahuri.

Athoni, B. K., Boodiand, I. H. and Guggari, A. K. (2016). Combining ability and heterosis for grain yield and its componets in pearl millet (Pennisetum glaucum (L) R. Br.). International Journal of Science and Nature,7(4): 786-794.

Badhe, P. L., Patil, H. T., Borole, D. N. and Thakare, S. M. (2018). Heterosis for yield and morpho-nutritional traits in pearl millet (Pennisetum glaucum L.). Electronic Journal of Plant Breeding, 9(2): $759-762$.

Bachkar, R.M., Pole, S.P. and Patil, S.N. (2014). Heterosis for grain yield and its components in pearl millet (Pennisetum glaucum L.). Indian J. of Dryland Agric. Res. \& Dev., 29 (1): 40-44.

Bhasker, K., Shashibhushan, D., Murali Krishna, K. and Bhave, M. H. V. (2017). Studies on heterosis for grain yield and its contributing characters in hybrids of pearl millet (Pennisetum glaucum (L) R. Br.). International Journal of Plant \& Soil Science, 18(5): 1-6.

Chittora, K. and Patel, J. A.(2017). Estimation of heterosis for grain yield and yield components in pearl millet (Pennisetum $\begin{array}{llll}\text { glaucum (L.) } & \text { R. } & \text { Br.). }\end{array}$ Int.J.Curr.Microbiol.App.Sci., $\quad$ 6(3): 412-418.

Karvar, S. H., Pawar, V. Y., and Patil, H. T. (2017). Heterosis and combining ability in pearl millet. Electronic Journal of Plant Breeding, 8 (4): 1197-1215.

Krishnan, Patel, M. R., Gami, M. S., Bhadauria, H. S. and Patel,Y. N. (2017). Genetic analysis in pearl millet 


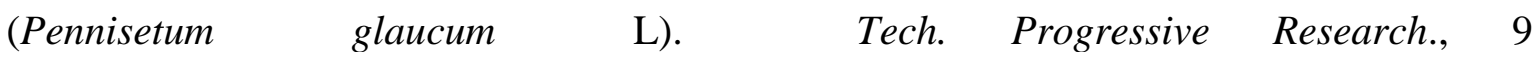
Int.J.Curr.Microbiol.App.Sc.», 6(11): 900-907.

Ladumor, V. L., Mungra, K. D. and Parmar, S. K. (2018). Heterosis for Grain Yield Components in Pearl Millet (Pennisetum glaucum (L) R. Br.). Trends in Biosciences, 11(27): 34733477.

Mungra, K.S., Dobariya, K.L., Babariya, C.A. and Sapovadiya M. H. (2014). Heterosis for grain yield and its components in pearl millet (Pennisetum glaucum (L) R. (Special): 643-646.

Patel, B. C., Doshi J. S. and Patel, J. A. (2016). Heterosis for grain yield components in pearl millet (Pennisetum glaucum L). Innovare Journal of Agricultural Science, 4(3): 1-3.

Pawar, V. Y., Kute, N. S., Patil, H. T., Awari, V. R., Gavali, R. K. and Deshmukh, G. P. (2015). Heterosis for earliness in pearl millet (Pennisetum glaucum (L) R. Br.). Bioinfolet., 12(3 B): 696-706.

Br.). Society for Sci. Dev. in Agric. and

\section{How to cite this article:}

Patil, S. H., P. B. Wadikar, A. R. Talape and Waghmode, S. L. 2021. Heterosis Analysis for Yield and its Components Traits in Pearl Millet [Pennisetum glaucum (L.) R. Br.]. Int.J.Curr.Microbiol.App.Sci. 10(01): 2392-2401. doi: https://doi.org/10.20546/ijcmas.2021.1001.278 